\title{
Talking About the Hidden in Architectural Education
}

The European Association of Architectural Education's annual conference of 2019 was held at the Faculty of Architecture in Zagreb from August 28th to 31st. Titled 'The Hidden School', it aimed to open a discussion on the substance and quality of architectural education, an architecture school's true character, the traits which - however explicitly or implicitly manifested - embody the school's culture and identity. The conference explored the subliminal quality of architectural education less apparent just by reading the curricula or following evaluation procedures, yet which represent a substantial quality or the culture of a school, quite clearly legible to those engaging in it. The invitation to explore this topic proposed five aspects of a school as triggers, focusing on tacit meanings situated between the lines of the syllabus, the spirit generated by students contributing to it or the educators personifying it, informal learning modalities, spaces it inhabits: the Educator, the Content, the Process, the Place, the Student. The scientific committee placed a question to the participating schools: "If the hidden school exists in parallel or as a background process, a self-generated search for fundamental answers, and its interpretation, manifestation or legibility has a multitude of facets, how can these aspects be captured?" Is it possible to assess the 'hidden'?

The topic of the conference emerged from discussions within EAAE's Education Academy, brought together by the motivating force of Johan de Walsche, on what we were talking about when talking about an architectural school, on what is measured and how it differs from what actually constitutes it. It was also an opportunity for introspection at the hosting school, the University of Zagreb's Faculty of Architecture, as the conference coincided with the centennial celebration of educating architects in Croatia and provided a forum to summarize what the school has built over a hundred years, but also open up discussions on where it is heading. Owing to the support and vision of EAAE president Oya Atalay Franck, the conference was also an opportunity to expand the reach of the association and open this discussion in a school itself hidden within the European context due to geography, economy and 
recent histories. The conference and its accompanying events structurally looked up to the resonant examples of recent EAAE annual conferences held in Milan (2015), Delft (2016), Bordeaux (2017) and Porto (2018), combining key-note lectures by educators and practitioners, a call for contributions inviting the community of the EAAE to reflect on the proposed topics, an international student workshop, field visits, research project disseminations and accompanying exhibitions: "Young Talent Architecture Award 2018" of the Mies van der Rohe Foundation, "re:EASA Rijeka 2018" of the European Architecture Students' Assembly, and "The Faculty of Architecture's School: A Brief Autobiography" - the exhibition marking the centennial of the hosting school. ${ }^{1}$

The conference was preceded by a self-organized student workshop exploring modalities of an ideal studio, called Course $X$ and led by Zagreb students Dora Gorenak, Filip Pračić, and Marin Nižić. Aiming to conceptualize an ideal course by examining the modalities of knowledge transmission, relations to the 'spirit ot time' as well as the discipline today, it took the shape of introspective self-analysis as a basis for exploring how knowledge and skills taught at schools relate to the moment as well as institutional frameworks. The public presentation of workshop results preceded the conference opening, offering a perspective on the 'hidden' explored from different cultural and educational backgrounds, outlining hypothetical modules and their implementation in various contexts, focusing among other things on process-driven courses embracing self-initiative, social engagement and intuition, empowering students in channeling and expressing their agency. To be able to establish such an environment, groups observed the supportive aspect of workshops, the desire to create an environment where connections are also based on caring, the necessity to move agency out of the institution, the need to shift importance from solution to problem statement and reaching out for feedback outside the immediate educational context.

Key-note lectures released insights related to the topic from various cultural backgrounds, inherited legacies, organizational and learning models, personal perspectives, research and practice. The conference was opened with an introductory provocation by Harriet Harriss, at the time of the conference newly appointed dean of the Pratt School of Architecture, focusing

1 Details of the program, summaries of exhibitions and events, as well as abstracts of all presented contributions, have been published in „The Hidden School - EAAE Annual Conference 2019, Zagreb: Book of Abstracts" (eds. Cavallo, R., Roth-Čerina, M.), ISBN 9789463661966 
on a fundamental topic of the need to decolonize the curriculum and face the background of the foundation of schools, the sources of their initial wealth, the bias in knowledge production as well as marginalization, in order to take a frank introspective look into the legacies we inherit and address them today. The first key-note speaker she introduced was Will Hunter, founder and director of the London School of Architecture, whose lecture's structure followed the conference's topics to explain the unique model of an architecture school organized as a network rather than hierarchical framework, operating through a series of relationships, using the city as a campus and source of acquiring complex knowledge, teaching in vacant spaces, working in cooperation with architectural practices, with students paying through earnings at offices, working in a collaborative environment and nurturing critical practice, aiming for a school as a heterarchy. His talk was followed by Lesley Lokko, who at that moment was moving from the position of director at the Graduate School of Architecture at University of Johannesburg in South Africa, a program she established, to assume the deanship of the Spitzer School of Architecture at New York's City College. By intertwining context and content, she bared the hidden, secret, subversive backgrounds, but also those of resistance, that made the pioneering Transformative pedagogies program one which enabled students to find their voice and express their architectural identity through research and work, providing an important step in the decolonization of higher education of the postcolonial South African environment. The ensuing discussion concluded on advocating for fluidity of schools - the more it shifts away from the 'inside'-'outside' dichotomy, the more relevance it gains.

The second day saw an intense mix of parallel sessions and key-note lectures, research project presentations and international workshops (the account of the European Architecture Student's Assembly hosted by EASA Croatia in Rijeka in 2018, and the concluding presentation of the Erasmus+ strategic partnership exploring thresholds in architectural education, the diploma studio and obligatory practice, titled Exploring the Field of Interaction in Architectural Design Education and concluding with a vernissage at the Museum of Architecture of the Croatian Academy of Sciences and Arts reflecting on the hosting school's centennial. Parallel sessions ran in three parallel streams on topics proposed by the conference call, further differentiating within the themes, but also zooming out in sessions discussing explorative strategies and reflecting on the hidden. The key-note lecture by Maruša Zorec, architect and professor at the Faculty of Architecture in Ljubljana, entered into a personal explication of the essence of our profession, 
and how it translates into what we teach. Viewing teaching and practice as inextricably linked and very personal, she provided a glimpse into the specific educational culture of her school, comprised of many schools made up by vertical studios whose identities are shaped by the leading mentor, relating subjects of interest - beyond the program or time we find ourselves in - to her exhibition at the Venice Architecture Biennale in 2018 titled 'Unveiling the Hidden'. Momoyo Kaijima, founder of Bow-Wow and professor at ETH, expanded on her Architectural behaviorology program as a means of accessing local resources, studying typology flux through analytical drawing as an educational tool, and how this ethnographical research can be utilized as a means of communication to improve concrete situations, transposed into design-build workshops. The hidden aspect of this process is the implicit de-schooling, engaging in self-creating learning resources to architecturally understand life, scape and their interactions.

The third day informed the audience on the many activities undertaken within EAAE's working groups: Education Academy, Research Academy and Conservation Network, before the final key-note lecture proposed in duet by An Fonteyn, architect at noAarchitecten and professor at ETH, and her student at ETH, Sara Sherif. They gave a most intimate look into the dialogue between teacher and student, and how it shifts shape and content, moving out of the immediate context of the school into travel, workshops, or working with other media. The lecture was a direct recount of letters the two exchanged from various points of departure, touching on many of the conference's topics in a most poetic manner. It shed light on the interactions between student and teacher situated in-between, not explicit in the brief nor the formal end of an educational module, evolving through time spent together, situated within a multitude of coordinates: geographical, inherited, points of reference brought in as a personal atlas which mutually grows through this exchange.

The conference was concluded by a final discussion moderated by EAAE president Oya Atalay Franck and joined by guests representing the international circle of institutions bonded by efforts to keep the discipline of architecture - architectural education, research and practice - interconnected and engaged: Thomas Vonier, president of the International Union of Architects UIA; Georg Pendl, president of the Architects' Council of Europe ACE; Rashida Ng, president of the Board of Directors of the Association of Collegiate Schools of Architecture ACSA; Hazem Rashed-Ali, president of the Architectural Research Centers Consortium ARCC; Don Gray, Chair of the 
Standing Conference of Heads of Schools of Architecture SCHOSA; and final speakers An Fonteyne, professor at ETH Zurich and architect at NoAarchitecten with Sara Sherif, student at ETH. They reflected on the conference theme, triggered by the notion of 'inside' and 'outside' of the educational environment, mentioned or implied several times, making it clear this border should be blurred. The limit of the reach of a school is not in its physical boundaries, but now more than ever in its mental or identity boundaries. As they pointed out, a school should be of the society, integrated into it, and only then does it sustain its relevance - a school cannot exist on its own, independent of the societal or political context it operates in. When speaking of the profession itself, one can perhaps talk of an 'inside' as practice and the 'outside', pertaining to a struggle for significance within contemporary conditions of operation, or an effort to maintain idealism: architects on a constant quest for relevance and impact. The profession has a challenge to demonstrate how our skills are relevant for life on the planet, so how we learn to effectively engage and influence is as much a part of our upbringing as is the knowledge. The importance of the school being an active participant in its environment was therefore stressed, encouraging students to take a role and get involved in culture and society, lessening the distance of what is on the inside of a school and the outside world. However, the safe intimacy of a school must be protected to allow a boldness of topics in which everything can be questioned. This safe environment allows for failure as well - of the student as much as the teacher: the right to be wrong, to experiment without an imperative of excellence, bringing the discussion to the question of metrics and how we actually value or identify success. The school as platform for experimentation is beneficial to practice as well, and no barrier between the two should exist - fostering the relationship between education and practice does not just advance education, but also nurtures the reflective practitioner. Opportunities and tools for a shift in practice can start within a school, where problems are subjected to an abstraction: one acquires an approach, tools to tackle the unknown. More and more does the role from architect extend to architecturally thinking strategist, one with a deep understanding of the contemporary condition. This makes the importance of continuing joint efforts in research and discussions across the EU and internationally as valuable as ever, maintaining the conversation and establishing unity.

Finally, the discussion also, quite personally, opened the notion of hidden communications - the pervasive artificial stance of a student after entering a school, speaking in a new artificial language, clouding direct exchange between student 
and teacher, leaving a lot unsaid. As one of the workshop participants, Petronela Shredlova, wrapped up: teachers talk among themselves on how to improve education, but students should be invited into the discussion more often, allowed to help and openly reflect. Looking within, many aspects are perhaps not hidden, just silent. A direct conversation within the school, as much as among the school and environment, would advance dissolving boundaries.

$\star * *$

While looking back on the topic of the 2019 conference and putting this book of contributions together in a time of unprecedented change to many of the aspects we took as constant, one had to re-examine the starting hypothesis. The attempt to explore the less apparent aspects of architectural education still rested upon the belief that we have a school set in a physical space and that the nuances of interactions or processes take the form of verbal as much as non-verbal interaction. Compressing and flattening these interactions into a zoom/teams/ meet screen opened up a new array of possible explorations, but also greatly affected many of the aspects and examples of the hidden discussed in this book. How does a devoted educator interact with students today, what medium does the informal in-between of a workshop or a studio shift into, how does a school relate to the virtual public space? Beside the explored aspects taking on new meaning, the implosion of the external into private space opened new facets of the hidden which are yet to be examined, and the questions our planet faced provoked an acute need to address issues of contemporary society and our environment. Taking the opportunity to invite the reader to stay in touch in these further discussions, we see this publication as marking a conclusion as much as a new start.

Mia Roth-Čerina and Roberto Cavallo 



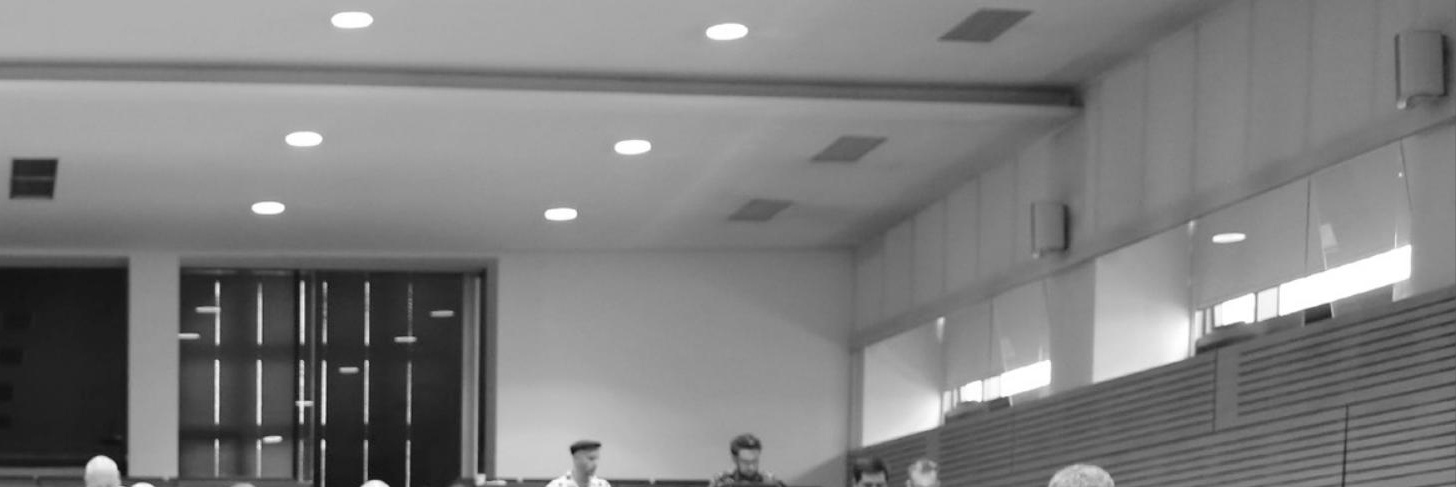

an

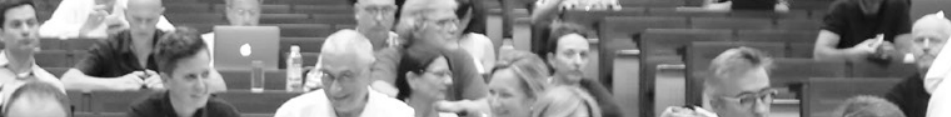
(1) $-1, \Rightarrow 31$ 